\title{
Revision and Conditional Inference for Abstract Dialectical Frameworks
}

\author{
Jesse Heyninck $^{1,2}$ ， Gabriele Kern-Isberner ${ }^{1}$, Tjitze Rienstra ${ }^{3}$, Kenneth Skiba ${ }^{3}$, Matthias \\ Thimm $^{3}$ \\ ${ }^{1}$ Technische Universität Dortmund, Dortmund, Germany \\ ${ }^{2}$ University of Cape Town and CAIR, South-Africa \\ ${ }^{3}$ University of Koblenz-Landau, Koblenz, Germany \\ \{jesse.heyninck, gabriele.kern-isberner\}@tu-dortmund.de, \\ \{thimm, kennethskiba,rienstra\}@uni-koblenz.de
}

\begin{abstract}
For propositional beliefs, there are well-established connections between belief revision, defeasible conditionals and nonmonotonic inference. In argumentative contexts, such connections have not yet been investigated. On the one hand, the exact relationship between formal argumentation and nonmonotonic inference relations is a research topic that keeps on eluding researchers despite recently intensified efforts, whereas argumentative revision has been studied in numerous works during recent years. In this paper, we show that similar relationships between belief revision, defeasible conditionals and nonmonotonic inference hold in argumentative contexts as well. We first define revision operators for abstract dialectical frameworks, and use such revision operators to define dynamic conditionals by means of the Ramsey test. We show that such conditionals can be equivalently defined using a total preorder over three-valued interpretations, and study the inferential behaviour of the resulting conditional inference relations.
\end{abstract}

\section{Introduction}

Belief revision, defeasible conditionals and nonmonotonic inference relations form a triangle of strongly connected concepts within knowledge representation. Conditionals (Nute 1984) have been a cause of concern for philosophers for the better part of the history of philosophy, but within the formal logical study of conditionals, in the last semi-century, a lot of progress has been made. A central idea in the study of conditionals is that in the evaluation of a conditional "if $\phi$ then $\psi$ " (formally, $(\psi \mid \phi)$ ), it suffices to check for the validity of $\psi$ in a certain subset of all models of $\phi$. This is often modelled using a selection function over the set of possible worlds $\Omega: f: \Omega \times \wp(\Omega) \rightarrow \wp(\Omega)$. A conditional $(\psi \mid \phi)$ is then true at a world $\omega$ according to a selection function $f$ iff every world in $f(\omega,[\phi])$ validates $\psi$. Nonmonotonic inference relations (Kraus, Lehmann, and Magidor 1990; Shoham 1987), on the other hand, have been studied semantically using a preference relation $\preceq$ over the set of possible worlds. A nonmonotonic inference $\phi \sim \psi$ is then valid iff $\psi$ holds in all $\preceq$-minimal $\phi$-worlds. The relations between conditionals and nonmonotonic inference relations are clear, then, as $\min \prec$ can be viewed as a selection function. As such, a conditional inference relation $\sim$ can be associated with a nonmotonic inference relation s.t. $\preceq \sim(\psi \mid \phi)$ iff $\phi \sim \psi$. Belief revision studies the effect of the dynamics of propositional beliefs, and the consolidation of belief revision as a field of study is often identified with the formulation of the AGM-theory (Alchourrón, Gärdenfors, and Makinson 1985) of belief revision. Close relationships between belief revision and conditional logics were noticed by means of the Ramsey test (Ramsey 1931), which also gave rise to impossibility results on the compatibility of belief revision and conditional reasoning (Gärdenfors 1986). However, when (Katsuno and Mendelzon 1991) showed that total preorders underlie AGM-belief revision in a fundamental and inevitable way, it was at once also established that belief revision, conditional logic and nonmonotonic inference were shown to be fully compatible. They can thus be seen as three different sides of a single topic or mode of reasoning (Gärdenfors 1990; Makinson 1993), at least when restricted to propositional beliefs. Indeed, when moving to other kinds of belief revision (e.g. (Hansson 1999; Delgrande and Peppas 2015)), weaker kinds of conditionals (Hawthorne 2007; Makinson 2011) or other forms of nonmonotonic inference, these interrelations tend to break down.

Another important field in knowledge representation is formal argumentation. Argumentative reasoning is usually perceived as a specific form of nonmonotonic reasoning (see e.g. (Rienstra 2014; Booth et al. 2012; Booth et al. 2013)) but attempts to transform reasoning systems from one side into systems of the other side have been revealing gaps that could not be closed (cf., e.g., (Thimm and Kern-Isberner 2008; Kern-Isberner and Simari 2011; Heyninck 2019)). Therefore, in spite of the abundance of existing work studying connections between the two fields, a general way of obtaining well-behaved non-monotonic conditional inference relations on the basis of argumentative contexts has not been defined yet. On the other hand, belief dynamics in general and belief revision in particular has been studied intensively for formal argumentation. Therefore, in this paper we make a systematic and general attempt to answer the question as to whether belief revision, nonmonotonic inference relations and defeasible conditionals form an interconnected triangle in an argumentative context as they do in a propositional setting. We answer this question for Abstract Dialectical Frameworks (ADFs) (Brewka et al. 2013), an approach to formal argumentation, which subsumes many other argumentative formalisms in a generic, 


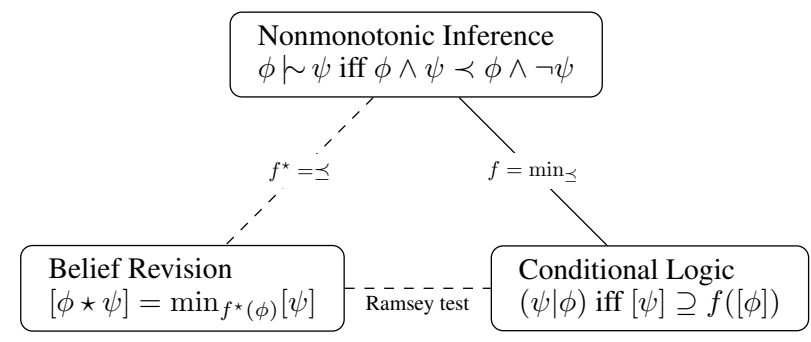

Figure 1: Graphical Representation of connections between belief revision, nonmonotonic inference and conditional logics. A full line means there is a full correspondence between the two concepts, whereas a dashed line means that there is additional information needed for a full correspondence. E.g. to define a belief revision operator on the basis of a nonmonotonic inference relation one needs to additionally assume a context $K$ which corresponds to $\phi$, see e.g. (Makinson and Gärdenfors 1991).

logic-based way.

In this paper, we investigate connections between belief revision, nonmonotonic inference and defeasible conditionals within abstract dialectical argumentation. We first define and study revision of ADFs in depth and then use these revisions to define conditional inference for ADFs. Then, we define dynamic nonmonotonic inference relations based on the Ramsey test (Ramsey 1931). We study these inference relations in terms of rationality postulates known from defeasible conditionals. We accordingly summarize the contributions of this paper as follows: (1) definition of belief revision of ADFs by formulas, (2) a semantical characterisation of such revision operators in terms of total preorders over three-valued interpretations, (3) the definition of $d y$ namic conditional inference relations for ADFs based on the Ramsey test, and (4) a study of dynamic conditional inference relations in terms of postulates known from defeasible conditionals.

Outline of this Paper: We first state all the necessary preliminaries in Section 2 on propositional logic (Section 2.1), three-valued logic (Section 2.2), reasoning with nonmonotonic conditionals (Section 2.3), propositional revision (Section 2.4) and abstract dialectical argumentation (Section 2.5). We then define revision of ADFs under various semantics, in particular under the preferred semantics (Section 3.3) and the grounded semantics (Section 3.4). Thereafter, in Section 4, we define and study dynamic conditionals based on such revisions. We compare our approach with related work in Section 5.

\section{Preliminaries}

In the following, we briefly recall some general preliminaries on propositional logic, as well as technical details on conditional logic and ADFs (Brewka et al. 2013).

\subsection{Propositional Logic}

For a set At of atoms let $\mathcal{L}(\mathrm{At})$ be the corresponding propositional language constructed using the usual connectives $\wedge$ (and), $\vee($ or $), \neg$ (negation) and $\rightarrow$ (material implication). A (classical) interpretation (also called possible world) $\omega$ for a propositional language $\mathcal{L}(\mathrm{At})$ is a function $\omega:$ At $\rightarrow\{\mathrm{T}, \mathrm{F}\}$. Let $\Omega(\mathrm{At})$ denote the set of all interpretations for At. We simply write $\Omega$ if the set of atoms is implicitly given. An interpretation $\omega$ satisfies (or is a model of) an atom $a \in$ At, denoted by $\omega \models a$, if and only if $\omega(a)=\mathrm{T}$. The satisfaction relation $\models$ is extended to formulas as usual. As an abbreviation we sometimes identify an interpretation $\omega$ with its complete conjunction, i. e., if $a_{1}, \ldots, a_{n} \in$ At are those atoms that are assigned $\mathrm{T}$ by $\omega$ and $a_{n+1}, \ldots, a_{m} \in$ At are those propositions that are assigned $\mathrm{F}$ by $\omega$ we identify $\omega$ by $a_{1} \ldots a_{n} \overline{a_{n+1}} \ldots \overline{a_{m}}$ (or any permutation of this). For example, the interpretation $\omega_{1}$ on $\{a, b, c\}$ with $\omega(a)=\omega(c)=\mathrm{T}$ and $\omega(b)=\mathrm{F}$ is abbreviated by $a \bar{b} c$. For $\Phi \subseteq \mathcal{L}($ At) we also define $\omega \models \Phi$ if and only if $\omega \models \phi$ for every $\phi \in \Phi$. Define the set of models $\operatorname{Mod}(X)=\{\omega \in \Omega(\mathrm{At})|\omega|=X\}$ for every formula or set of formulas $X$. A formula or set of formulas $X_{1}$ entails another formula or set of formulas $X_{2}$, denoted by $X_{1} \vdash X_{2}$, if $\operatorname{Mod}\left(X_{1}\right) \subseteq \operatorname{Mod}\left(X_{2}\right)$.

\subsection{Kleenes Three-Valued Logic}

A 3-valued interpretation for a set of atoms At is a function $v: S \rightarrow\{\top, \perp, u\}$, which assigns to each atom in At either the value $\top$ (true, accepted), $\perp$ (false, rejected), or $u$ (unknown). The set of all three-valued interpretations for a set of atoms At is denoted by $\mathcal{V}(A t)$. We sometimes denote an interpretation $v \in \mathcal{V}\left(\left\{x_{1}, \ldots, x_{n}\right\}\right)$ by $\dagger_{1} \ldots \dagger_{n}$ with $v\left(x_{i}\right)=\dagger_{i}$ and $\dagger_{i} \in\{\top, \perp, u\}$, e.g. $T \top$ denotes $v(a)=v(b)=\top$. A 3-valued interpretation $v$ can be extended to arbitrary propositional formulas $\phi \in \mathcal{L}(\mathrm{At})$ via the truth tables in Table 1. We furthermore extend the language with a second, weak negation $\sim$, which is evaluated to true if there is no positive information for the negated formula (i.e. the negated formula is false or undecided) and thus expresses non-truth, as opposed to $\neg$ which expresses explicit falsity. The truth table for $\sim$ can also be found in Table $1 .{ }^{1}$ It will prove convenient to define the connective $\odot$ which stipulates a formula is undecided. We define $\odot \phi=\sim(\neg \phi \vee \phi)$. We define $\mathcal{L}^{\mathrm{K}}(\mathrm{At})$ as the language based on At, the unary connectives $\langle\neg, \sim, \odot\rangle$ and the binary connectives $\langle\wedge, \vee, \rightarrow\rangle$.

We can show that $\odot$ expresses the undecidedness of any formula $\phi \in \mathcal{L}^{\mathrm{K}}$ :

Fact 1. For any $\phi \in \mathcal{L}^{\mathrm{K}}(\mathrm{At}), v(\odot \phi)=\top$ iff $v(\phi)=u$.

The following facts about $\sim$ will prove useful below:

Fact 2. For any $\phi \in \mathcal{L}^{\mathrm{K}}(\mathrm{At})$ and any $v \in \mathcal{V}(\mathrm{At})$ : (1) $v(\sim \phi) \neq u$, and $(2) v(\sim \sim \phi)=\top$ iff $v(\phi)=\top$.

We define the set of three-valued interpretations that satisfy a formula $\phi \in \mathcal{L}^{\mathrm{K}}(\mathrm{At})$ as $\mathcal{V}(\phi)=\{v \in \mathcal{V}(\mathrm{At}) \mid v(\phi)=$ Т \}. A formula $X_{1} \mathrm{~K}$-entails another formula $X_{2}$, denoted $X_{1} \vdash_{\mathrm{K}} X_{2}$, if $\mathcal{V}\left(X_{1}\right) \subseteq \mathcal{V}\left(X_{2}\right) . X_{1} \equiv_{\mathrm{K}} X_{2}$ iff $X_{1} \vdash_{\mathrm{K}} X_{2}$ and $X_{2} \vdash{ }_{\mathrm{K}} X_{1}$.

\footnotetext{
${ }^{1}$ In the terminology of (Urquhart 2001), the negation $\sim$ corresponds to Bochvar's external negation (Bochvar and Bergmann 1981) and $\neg$ corresponds to Kleene's negation in his three-valued logic. $\sim$ is also called Kleene's weak negation (Varzi and Warglien 2003), since the conditions for $\sim \phi$ being satisfied are weaker than those for $\neg \phi$ being satisfied (i.e. $\{\neg \phi\} \vdash_{\mathrm{K}} \sim \phi$ ).
} 


\begin{tabular}{c||c|c|cc||cccc||ccc} 
& $\neg$ & $\sim$ & $\odot$ & $\wedge$ & $\top$ & $u$ & $\perp$ & $\vee$ & $\top$ & $u$ & $\perp$ \\
\hline$\top$ & $\perp$ & $\perp$ & $\perp$ & $\top$ & $\top$ & $u$ & $\perp$ & $\top$ & $\top$ & $\top$ & $\top$ \\
$u$ & $u$ & $\top$ & $\top$ & $u$ & $u$ & $u$ & $\perp$ & $u$ & $\top$ & $u$ & $u$ \\
$\perp$ & $\top$ & $\top$ & $\perp$ & $\perp$ & $\perp$ & $\perp$ & $\perp$ & $\perp$ & $\top$ & $u$ & $\perp$
\end{tabular}

Table 1: Truth-tables for connectives in Kleene's K

Given an interpretation $v \in \mathcal{V}(\mathrm{At})$, we define:

$$
\text { form }(v)=\bigwedge_{v(a)=\top} a \wedge \bigwedge_{v(a)=\perp} \neg a \wedge \bigwedge_{v(a)=u} \odot a
$$

Clearly, form $(v)$ expresses exactly the beliefs expressed by a three-valued interpretations:

Fact 3. For any $v \in \mathcal{V}\left(\right.$ At) and any $a \in$ At: (1) form $(v) \vdash_{\mathrm{K}}$ $a$ iff $v(a)=\top$; (2) form $(v) \vdash_{\mathrm{K}} \neg a$ iff $v(a)=\perp$; (3) form $(v) \vdash_{\mathrm{K}} \odot a$ iff $v(a)=u$.

\subsection{Defeasible Inference and Nonmonotonic Conditionals}

There are many different conditional logics (cf., e.g., (Kraus, Lehmann, and Magidor 1990; Nute 1984)), but a common idea underlying many semantics for nonmonotonic conditionals is that to validate the acceptance of a conditional $(\psi \mid \phi)$, it suffices to look whether its material counterpart $\phi \rightarrow \psi$ is validated in a subset of possible worlds. In this work, we will assume that a preorder $\preceq \subseteq \wp(\Omega(A t) \times$ $\Omega(\mathrm{At}))$ over the set of possible worlds can be used to encode relevance of the possible worlds w.r.t. evaluation of conditionals. In more detail, we will state that a conditional $(\psi \mid \phi)$ is accepted in a context encoded by $\preceq$ iff the consequent is validated by all $\preceq$-minimal worlds models of the antecedent $\phi$, in symbols:

$$
\operatorname{Mod}(\psi) \supseteq \min _{\preceq}(\operatorname{Mod}(\phi))
$$

This is in full compliance with defeasible inference relations $\phi \sim \psi$ (Makinson 1988) expressing that from $\phi, \psi$ may be plausibly/defeasibly derived. We say that $\phi \preceq \psi$ iff $\omega \preceq \omega^{\prime}$ for some $\omega \in \min _{\preceq}(\operatorname{Mod}(\phi))$ and some $\omega^{\prime} \in \min _{\preceq}(\operatorname{Mod}(\psi))$. This allows for expressing the validity of defeasible inferences via stating that $\phi \sim_{\prec} \psi$ iff $(\phi \wedge \psi) \prec(\phi \wedge \neg \psi)$. Thus, nonmonotonic conditionals as defined above can be seen as a syntactic counterpart to defeasible inference, in the sense that $(\psi \mid \phi)$ is accepted in a context encoded by $\preceq$ iff $\phi \sim_{\preceq} \psi$

Example 1. Consider $\preceq$ defined over $\Omega(\{a, b, c\})$ as follows:

$a b c, \quad a b \bar{c}, \quad a \bar{b} c, \quad \bar{a} b c \quad \prec \quad a \bar{b} \bar{c}, \quad \bar{a} b \bar{c}, \quad \bar{a} \bar{b} c, \quad \bar{a} \bar{b} \bar{c}$ Thus, for example, $\neg a \sim_{\preceq} b, \neg b \sim_{\preceq} a, \neg a \vee \neg b \sim_{\preceq} c$, $\top \sim_{\preceq} a \vee b$ and $a K \preceq c$.

We recall some properties of conditional consequence relations (Kraus, Lehmann, and Magidor 1990):

(REF) $\quad \phi \in \mathcal{L}(\mathrm{At})$ implies $\quad \phi \sim \phi$

(CUT) $\phi \sim \psi$ and $\phi \wedge \psi \sim \gamma$ imply $\phi \sim \gamma$

(CM) $\quad \phi \sim \psi$ and $\phi \mid \sim \gamma \quad$ imply $\quad \phi \wedge \psi \sim \gamma$

(RW) $\phi \sim \psi$ and $\psi \models \gamma \quad$ imply $\quad \phi \sim \gamma$

(LLE) $\phi \equiv \psi$ and $\psi \sim \sim \gamma \quad$ imply $\quad \phi \sim \gamma$

(OR) $\quad \phi \sim \gamma \quad$ and $\quad \psi \sim \gamma \quad$ imply $\quad(\phi \vee \psi) \sim \gamma$

(RM) $\quad \phi \sim \gamma$ and $\phi K \neg \psi$ imply $\quad \phi \wedge \psi \sim \gamma$
Defeasible inference relations $\sim_{\preceq}$ based on total preorders $\preceq$ satisfy these properties:

Proposition 1 ((Makinson 1993)). For any total preorder $\preceq$, $\sim \prec$ satisfies (REF), (CUT), (CM), (RW), (LLE), (OR) and $(\mathrm{RM})$.

\subsection{Revising Propositional Formulas}

We now recall the so-called AGM-approach to belief revision (Alchourrón, Gärdenfors, and Makinson 1985) as reformulated for propositional formulas by (Katsuno and Mendelzon 1991). The following postulates for revision operators $\star: \mathcal{L} \times \mathcal{L} \rightarrow \mathcal{L}$ are formulated:

(R1) $\phi \star \psi \vdash \psi$

(R2) If $\phi \wedge \psi$ is satisfiable, then $\phi \star \psi \equiv \psi \wedge \phi$

(R3) If $\psi$ is satisfiable, then so is $\phi \star \psi$

(R4) If $\phi_{1} \equiv \phi_{2}$ and $\psi_{1} \equiv \psi_{2}, \phi_{1} \star \psi_{1} \equiv \phi_{2} \star \psi_{2}$

(R5) $\quad(\phi \star \psi) \wedge \mu \vdash \phi \star(\psi \wedge \mu)$

(R6) If $(\phi \star \psi) \wedge \mu$ is satisfiable, then $\phi \star(\psi \wedge \mu) \vdash$ $(\phi \star \psi) \wedge \mu$

An important result is the semantical characterisation of such a belief revision operator. For such a characterisation, a function $f: \mathcal{L}(\mathrm{At}) \rightarrow \wp(\Omega(\mathrm{At}) \times \Omega(\mathrm{At}))$ that assigns to each propositional formula $\phi \in \mathcal{L}$ a preorder $\preceq_{\phi}$ over $\Omega(\mathrm{At})$.

Definition 1 ((Katsuno and Mendelzon 1991)). Given a formula $\phi \in \mathcal{L}($ At $)$, a function $f: \mathcal{L}($ At $) \rightarrow \wp(\Omega(A t) \times \Omega(A t))$ assigning preorders $\preceq_{\phi}$ over $\Omega($ At $)$ to every formula $\phi \in$ $\mathcal{L}(\mathrm{At})$ is faithful iff:

1. For every $\phi \in \mathcal{L}($ At $)$, if $\omega, \omega^{\prime} \in \operatorname{Mod}(\phi)$ then $\omega \nprec_{\phi} \omega^{\prime}$,

2. For every $\phi \in \mathcal{L}(\mathrm{At})$, if $\omega \in \operatorname{Mod}(\phi)$ and $\omega^{\prime} \notin \operatorname{Mod}(\phi)$ then $\omega \preceq_{\phi} \omega^{\prime}$,

3. For every $\phi, \phi^{\prime} \in \mathcal{L}(\mathrm{At})$, if $\phi \equiv \phi^{\prime}$ then $\preceq_{\phi}=\preceq_{\phi^{\prime}}$.

In (Katsuno and Mendelzon 1991) the following representation theorem for an AGM revision operator $\star$ was shown:

Theorem 1 ((Katsuno and Mendelzon 1991)). An operator $\star: \mathcal{L}(\mathrm{At}) \times \mathcal{L}(\mathrm{At}) \rightarrow \mathcal{L}(\mathrm{At})$ is a revision operator iff there exists a faithful mapping $f^{\star}: \mathcal{L}(\mathrm{At}) \rightarrow \wp(\Omega(\mathrm{At}) \times \Omega(\mathrm{At}))$ that maps each formula $\phi \in \mathcal{L}(\mathrm{At})$ to a total preorder s.t.:

$$
\operatorname{Mod}(\phi \star \psi)=\min _{f^{\star}(\phi)}(\operatorname{Mod}(\psi))
$$

\subsection{Abstract Dialectical Frameworks}

We briefly recall some technical details on ADFs following loosely the notation from (Brewka et al. 2013). An ADF $D$ is a tuple $D=(\mathrm{At}, L, C)$ where At is a finite set of atoms, $L \subseteq$ At $\times$ At is a set of links, and $C=\left\{C_{s}\right\}_{s \in \text { At }}$ is a set of total functions $C_{s}: 2^{\text {par }_{D}(\mathrm{At})} \rightarrow\{\top, \perp\}$ for each $s \in \mathrm{At}$ with $\operatorname{par}_{D}(s)=\left\{s^{\prime} \in\right.$ At $\left.\mid\left(s^{\prime}, s\right) \in L\right\}$ (also called acceptance functions). An acceptance function $C_{s}$ defines the cases when the statement $s$ can be accepted (truth value $T$ ), depending on the acceptance status of its parents in $D$. By abuse of notation, we will often identify an acceptance function $C_{s}$ by its equivalent acceptance condition which models the acceptable cases as a propositional formula. $\mathfrak{D}(\mathrm{At})$ denotes the set of all ADFs $D=($ At $, L, C)$. 
Example 2. We consider the following ADF $D_{1}=$ $(\{a, b, c\}, L, C)$ with $L=\{(a, b),(b, a),(a, c),(b, c)\}$ and

$$
C_{a}=\neg b \quad C_{b}=\neg a \quad C_{c}=\neg a \vee \neg b
$$

Informally, the acceptance conditions can be read as " $a$ is accepted if $b$ is not accepted", " $b$ is accepted if $a$ is not accepted" and " $c$ is accepted if $a$ is not accepted or $b$ is not accepted".

An ADF $D=($ At $, L, C)$ is interpreted through 3-valued interpretations $\mathcal{V}(\mathrm{At})$. Recall that $\Omega(\mathrm{At})$ consists of all the two-valued interpretations (i.e. interpretations such that for every $s \in$ At, $v(s) \in\{\top, \perp\})$. We define the information order $\leq_{i}$ over $\{T, \perp, u\}$ by making $u$ the minimal element: $u<_{i} T$ and $u<_{i} \perp$ and this order is lifted pointwise as follows (given two valuations $v, w$ over At): $v \leq_{i} w$ iff $v(s) \leq_{i} w(s)$ for every $s \in$ At. The set of two-valued interpretations extending a valuation $v$ is defined as $[v]^{2}=$ $\left\{w \in \Omega(\mathrm{At}) \mid v \leq_{i} w\right\}$. Given a set of valuations $V$, the consensus operator $\Pi_{i}$ is defined as: $\Pi_{i} V(s)=v(s)$ if for every $v^{\prime} \in V, v(s)=v^{\prime}(s)$ and $\Pi_{i} V(s)=u$ otherwise. $\Gamma_{D}(v):$ At $\rightarrow\{\top, \perp, u\}$ where $s \rightarrow \Pi_{i}\left\{w\left(C_{s}\right) \mid w \in\right.$ $\left.[v]^{2}\right\}$. Thus, $\Gamma_{D}(v)$ assigns to $s$ the truth-value that all twovalued extensions of $v$ assign to the condition $C_{s}$ of $s$, if they agree on $C_{s}$, and $\mathrm{U}$ otherwise.

Definition 2. Let $D=($ At $, L, C)$ be an ADF with $v:$ At $\rightarrow$ $\{\top, \perp, u\}$ an interpretation:

- $v$ is admissible for $D$ iff $v \leq_{i} \Gamma_{D}(v)$.

- $v$ is complete for $D$ iff $v=\Gamma_{D}(v)$.

- $v$ is preferred for $D$ iff $v$ is $\leq_{i}$-maximally complete.

- $v$ is grounded for $D$ iff $v$ is $\leq_{i}$-minimally complete.

We denote by admissible, complete $(D), \operatorname{prf}(D)$, respectively grounded $(D)$ the sets of complete, preferred, grounded respectively interpretations of $D$.

We finally define consequence relations for ADFs:

Definition 3. Given Sem $\in\{$ prf, grounded $\}$, an ADF $D=$ $($ At $, L, C)$ and $\phi \in \mathcal{L}^{\mathrm{K}}(\mathrm{At})$ we define: $D \sim_{\text {Sem }}^{n} \phi$ iff $v(\phi)=$ $\top[\perp]$ for all $v \in \operatorname{Sem}(D)$.

Example 3 (Example 2 continued). The ADF of Example 2 has three complete models $v_{1}, v_{2}, v_{3}$ with:

$$
\begin{array}{lll}
v_{1}(a)=\top & v_{1}(b)=\perp & v_{1}(c)=\top \\
v_{2}(a)=\perp & v_{2}(b)=\top & v_{2}(c)=\top \\
v_{3}(a)=u & v_{3}(b)=u & v_{3}(c)=u
\end{array}
$$

$v_{3}$ is the grounded interpretation whereas $v_{1}$ and $v_{2}$ are both preferred.

It will be important to have characterisations of realizability of sets of interpretations under some semantics:

Definition 4. Given a set of atoms At, a set of interpretations $\mathcal{V} \subseteq \mathcal{V}(\mathrm{At})$ is realizable under semantics Sem iff there exists an ADF $D \in \mathfrak{D}($ At $)$ s.t. $\operatorname{Sem}(D)=\mathcal{V}$.

(Pührer 2020) shows that a set of interpretations is realizable under prf iff it is a $\leq_{i}$-anti-chain, whereas every (and only) singleton sets are realizable under grounded:

Proposition 2 ((Pührer 2020)). Given a set of atoms At, (1) a set of interpretations $\mathcal{V} \subseteq \mathcal{V}$ (At) is realizable under prf iff $\mathcal{V} \neq \emptyset$ and for every $v, v^{\prime} \in \mathcal{V}, v \mathbb{Z}_{i} v^{\prime}$ and $v^{\prime} \mathbb{Z}_{i} v$; (2) a set of interpretations $\mathcal{V} \subseteq \mathcal{V}(\mathrm{At})$ is realizable under grounded iff $\mathcal{V}$ has cardinality 1.

\section{ADF-revisions for Trivalent Semantics}

In this section, we study revision of ADFs by formulas under trivalent semantics, in particular the preferred and grounded semantics. We define in Section 3.1 postulates for revision operators under trivalent semantics, which we characterise in terms of total preorders over three-valued interpretations for the preferred semantics (Section 3.3) and the grounded semantics (Section 3.4).

\subsection{ADF-revision under Trivalent Semantics: Postulates and Semantics}

In this section we define a new approach to revision of ADFs for three-valued semantics. In more detail, we define an operator $\star$ that allows to revise an ADF (under some threevalued semantics) by a formula in the language $\mathcal{L}^{\mathrm{K}}{ }^{2}$ In other words, $\star: \mathfrak{D}(\mathrm{At}) \times \mathcal{L}^{\mathrm{K}}(\mathrm{At}) \rightarrow \mathfrak{D}(\mathrm{At})$. We adapt the AGM-postulates for propositional revision described in Section 2.4 to revision operators for ADFs in the following way:

Definition 5. An operator $\star$ is a trivalent ADF revision operator (in short, $\mathrm{ADF}_{\star}^{3}$-operator) for a semantics Sem iff $\star$ satisfies (for any $\phi, \psi, \mu \in \mathcal{L}^{\mathrm{K}}$ ):
$\left(\mathrm{ADF}_{\star}^{3} 1\right) \quad D \star \psi \sim_{\mathrm{Sem}}^{\cap} \psi .^{3}$
$\left(\mathrm{ADF}_{\star}^{3} 2\right) \quad$ If $\operatorname{Sem}(D) \cap \mathcal{V}(\psi) \neq \emptyset$ then $\operatorname{Sem}(D \star \psi)=$
$\begin{array}{ll}\left(\mathrm{ADF}_{\star}^{3} 3\right) & \operatorname{Sem}(D) \cap \mathcal{V}(\psi) . \\ \left(\mathrm{ADF}_{\star}(\psi) \neq \emptyset \text { then } \operatorname{Sem}(D \star \psi) \neq \emptyset .\right.\end{array}$
$\left(\mathrm{ADF}_{\star}^{3} 4\right) \quad$ If $\operatorname{Sem}(D)=\operatorname{Sem}\left(D^{\prime}\right)$ and $\psi \equiv_{\mathrm{K}} \psi^{\prime}$ then $\operatorname{Sem}(D \star \psi)=\operatorname{Sem}\left(D^{\prime} \star \psi^{\prime}\right)$.
$\left(\operatorname{ADF}_{\star}^{3} 5\right) \quad \operatorname{Sem}(D \star \psi) \cap \mathcal{V}(\mu) \subseteq \operatorname{Sem}(D \star(\psi \wedge \mu))$.
$\left(\operatorname{ADF}_{\star}^{3} 6\right) \quad$ If $\operatorname{Sem}(D \star \psi) \cap \mathcal{V}(\mu) \neq \emptyset$, then $\operatorname{Sem}(D \star$ $(\psi \wedge \mu)) \subseteq \operatorname{Sem}(D \star \psi) \cap \mathcal{V}(\mu)$.

We explain these postulates as follows: $\mathrm{ADF}_{\star}^{3} 1$ requires that the formula $\psi$ by which one revises is derivable in every Sem interpretation of the revised ADF. The second postulate $\mathrm{ADF}_{\star}^{3} 2$ can perhaps be better understood in its syntactical reformulation: If $D \nVdash \cap$ Sem $\sim \psi$, i.e. if $D$ has at least one Sem-interpretation that satisfies $\psi$, then $D \star \psi$ has as Sem-interpretations exactly the Seminterpretations of $D$ that satisfy $\psi \cdot \mathrm{ADF}_{\star}^{3} 3$ says that if we revise by a consistent formula, the resulting ADF will also admit Sem-interpretations. $A D_{\star}^{3} 4$ is a postulate of syntax-independence, which states that revising ADFs with the same Sem-interpretations by K-equivalent formulas results in Sem-equivalent revised ADFs. Finally, $\mathrm{ADF}_{\star}^{3} 5$ and $\mathrm{ADF}_{\star}^{3} 6$ are direct adaptions of the super-and sub-expansion postulates. They require, in the non-trivial case where $D \star \psi K \stackrel{\mathrm{Sem}}{\cap} \sim \mu$, that the Sem-interpretations of $D \star(\psi \wedge \mu)$ are exactly the Sem-interpretations of $D \star \psi$ that satisfy $\mu$.

The main question we answer in the rest of this section is whether $\mathrm{ADF}_{\star}^{3}$-operators can be characterised semantically analogously to propositional revision operators (Theorem 1). The central concept for such a characterisation will

\footnotetext{
${ }^{2}$ Recall, $\mathcal{L}^{\mathrm{K}}(\mathrm{At})$ is the language based on $\mathrm{At}$, the unary connectives $\langle\neg, \sim, \odot\rangle$ and the binary connectives $\langle\wedge, \vee, \rightarrow\rangle$

${ }^{3}$ Or, equivalently, $\operatorname{Sem}(D \star \psi) \subseteq \mathcal{V}(\psi)$.
} 
be that of a faithful mapping of ADFs to total preorders over $\mathcal{V}(\mathrm{At})$ :

Definition 6. Given a semantics Sem and an ADF $D=$ (At, $L, C$ ), a mapping $f: \mathfrak{D}(\mathrm{At}) \rightarrow \wp(\mathcal{V}(\mathrm{At}) \times \mathcal{V}(\mathrm{At}))$ associating a total preorder $\preceq_{D}$ to every ADF $D$ is a faithful mapping for semantics Sem if, for every $D \in \mathfrak{D}(\mathrm{At})$ and for every $v_{1}, v_{2} \in \mathcal{V}(\mathrm{At})$ :

1. if $v_{1} \in \operatorname{Sem}(D)$ then $v_{1} \preceq_{D} v_{2}$; and

2. if $v_{1} \in \operatorname{Sem}(D)$ and $v_{2} \notin \operatorname{Sem}(D)$ then $v_{1} \prec_{D} v_{2}$; and

3. if $\operatorname{Sem}(D)=\operatorname{Sem}\left(D^{\prime}\right)$ then $\preceq_{D}=\preceq_{D^{\prime}}$.

A faithful mapping is in general not sufficient to ensure a characterisation of $A D F_{\star}^{3}$-operators. The main problem is that a faithful mapping does not ensure that a selection of $\preceq_{D}$-minimal interpretations that satisfy $\phi$ are realizable by some ADF $D \star \phi \in \mathfrak{D}$ (At) under the semantics under consideration. In the folowing subsections, we investigate whether and how such realizability can be ensured by imposing additional conditions on faithul mappings. We shall see (in Section 3.2) that in general, such conditions cannot be found, by showing that for admissible and complete semantics no $\mathrm{ADF}_{\star}^{3}$-operator satisfying all postulates exists. Thereafter, we shall provide conditions and corresponding characterisation theorems for preferred (Section 3.3) and grounded (Section 3.4) semantics.

Remark 1. We have carried out a study similar to the one developed below for two-valued semantics such as the twovalued models and stable models. In view of space limitations, we merely remark here that the developments are entirely analogous to the development of revision under threevalue semantics: AGM-like revisions under a selected twovalued semantics can be characterized in terms of faithful mappings of ADFs to preorders, imposing additional conditions to ensure realizability of every selection of possible worlds.

\subsection{Impossibility of Rational Revision under Admissible and Complete Semantics}

In this section, we show that a revision operator that satisfies $\mathrm{ADF}_{\star}^{3} 1-\mathrm{ADF}_{\star}^{3} 6$ for the admissible or complete semantics does not exist. In particular, we show that no revision operator can satisfy $\mathrm{ADF}_{\star}^{3} 2$. A similar result can be found in (Diller et al. 2018, Proposition 2) for revision of abstract argumentation frameworks under complete semantics. Intuitively, the reason that no revision operator satisfying $\mathrm{ADF}_{\star}^{3} 2$ for these semantics exists is that not every subset of $\operatorname{Sem}(D)$ is realizable under Sem for Sem $\in$ \{complete, admissible\}. For example, a set not containing the interpretation that sets $v(s)=u$ for every $s \in$ At is not realizable under admissible semantics. Thus, if we revise $D$ by $\phi$ that is satisfied by exactly such a subset, $A F_{\star}^{3} 2$ forces $\operatorname{Sem}(D \star \phi)$ to equal a non-realizable set of interpretations.

Proposition 3. There is no operator $\star: \mathfrak{D}(\mathrm{At}) \times \mathcal{L}^{\mathrm{K}}(\mathrm{At}) \rightarrow$ $\mathfrak{D}(\mathrm{At})$ that satisfies $\mathrm{ADF}_{\star}^{3} 2$ for Sem $=$ complete or Sem $=$ admissible.
Proof. Suppose towards a contradiction that $\mathrm{ADF}_{\star}^{3} 2$ holds for an operator $\star$ for Sem $\in\{$ complete, admissible $\}$. Notice that complete $(D)=\{u, \top, \perp\}=\operatorname{admissible}(D)$. Consider the revision $D \star(a \vee \neg a)$. Since $\mathcal{V}(a \vee$ $\neg a)=\{\top, \perp\}, \mathcal{V}(a \vee \neg a) \cap \operatorname{Sem}(D) \neq \emptyset$ for Sem $\in$ \{complete, admissible\}, and thus, with our supposition that $\mathrm{ADF}_{\star}^{3} 2$ holds for $\star$ under Sem, $\operatorname{Sem}(D \star(a \vee \neg a))=$ $\operatorname{Sem}(D) \cap \mathcal{V}(a \vee \neg a)=\{\top, \perp\}$. But there is no ADF $D \star(a \vee \neg a) \in \mathfrak{D}(\{a\})$ s.t. $\operatorname{Sem}(D \star(a \vee \neg a))=\{\top, \perp\}$, i.e. the result of this revision is not realizable under Sem. To see this for Sem $=$ admissible, it suffices to observe that $u \in$ admissible $\left(D^{\prime}\right)$ for any $D^{\prime} \in \mathfrak{D}(\{a\})$. To see this for Sem $=$ complete, it suffices to observe that there exists for any ADF a unique $\leq_{i}$-minimal complete extension (Brewka et al. 2013). However, $\{T, \perp\}$ does not contain a unique $\leq_{i}$-minimal element.

\subsection{Revision of ADFs under Preferred Semantics}

In this section, we give a semantical characterisation of revision operators for preferred semantics, in terms of $i$-modular faithful mappings (imf-mappings). The following example shows that faithful mappings do not always lead to a sound semantical characterisation of $\mathrm{ADF}_{\star}^{3}$-revision operators for preferred semantics:

Example 4. We show that a naive adaption of Dalal's revision operator (Dalal 1988) does not lead to a well-defined revision operator. We use the symmetric distance function $\triangle$ defined between truth-values as follows: $T \triangle \perp=1$, $\top \triangle u=\perp \triangle u=0.5$ and $x \triangle x=0$ for any $x \in\{\top, \perp, u\}$ (cf. (Strass 2014)). We then lift this to interpretations $v, v^{\prime} \in$ $\mathcal{V}\left(\right.$ At) as follows: $v \triangle v^{\prime}=\Sigma_{s \in \text { At }} v(s) \triangle v^{\prime}(s)$. Defining a faithful preorder $\preceq_{D}$ based solely on this distance function (e.g. by setting $v_{1} \preceq_{D}^{\text {prf, } \Delta} v_{2}$ iff $\min _{v \in \operatorname{prf}(D)}\left(v \Delta v_{1}\right) \leq$ $\left.\min _{v \in \operatorname{prf}(D)}\left(v \Delta v_{2}\right)\right)$ would not result in an selection realizable under prf, since there could be $\preceq_{D}^{\text {prf, } \Delta}$-equal interpretations that are not $\leq_{i}$-incompatible.

Take e.g. the ADF $D_{1}$ from Example 2. Notice that $\operatorname{prf}(D)=\{\top \perp \top, \perp \top \top\}$ and $\top u u, \top \perp \perp \in$ $\min _{\preceq_{D}^{\text {prf }, \Delta}} \mathcal{V}(a \wedge \sim b \wedge \sim c)$. Revising $D$ with $a \wedge \sim b \wedge \sim c$ would thus result in an ADF $D_{1} \star a \wedge \sim b \wedge \sim c$ which has $T u u$ and $T \perp \perp$ among it's preferred extensions, which is impossible in view of Proposition 2, since Tuu and $T \perp \perp$ are not $\leq_{i}$-incompatible.

To avoid selections of interpretations that are nonrealizable under preferred semantics like in Example 4, an additional condition on faithful mappings has to be imposed. This condition we call $i$-modularity, and requires that every $\preceq_{D}$-layer is an $\leq_{i}$-antichain, i.e. all interpretations in a $\preceq_{D^{-}}$ layer are $\leq_{i}$-incompatible. We denote, for a preorder $\preceq$, $v \preceq v^{\prime}$ and $v^{\prime} \preceq v$ as $v \approx v^{\prime}$.

Definition 7. Given a semantics Sem and an ADF $D=$ $($ At $, L, C)$, a mapping $f: \mathfrak{D}(\mathrm{At}) \rightarrow \wp(\mathcal{V}(\mathrm{At}) \times \mathcal{V}(\mathrm{At}))$ associating a total preorder $\preceq_{D}$ to every ADF $D$ is an $i$ modular faithful mapping (imf-mapping) for semantics Sem if it is faithful w.r.t. Sem and for every $D \in \mathfrak{D}(\mathrm{At})$ and every $v_{1}, v_{2} \in \mathcal{V}(\mathrm{At})$ : if $v_{1} \approx_{D} v_{2}$ then $v_{1} \nless_{i} v_{2}$ and $v_{2} \nless_{i} v_{1}$. 
In Theorem 2 we show that $\mathrm{ADF}_{\star}^{3}$-operators for the preferred semantics can be characterized by imf-mappings. The proof of this theorem is analogous to the proof of the similar theorem for propositional belief revision in (Katsuno and Mendelzon 1991), with two exceptions: (1) every mention of propositional logic respectively possible worlds is substituted by Kleene's three-valued logic respectively threevalued interpretations and (2) realizability of $D \star \phi$ has to be accounted for and is shown to correspond to the requirement of i-modularity.

Theorem 2. ${ }^{4} \star: \mathfrak{D}(\mathrm{At}) \times \mathcal{L}^{\mathrm{K}}(\mathrm{At}) \rightarrow \mathfrak{D}(\mathrm{At})$ is a trivalent ADF revision operator $\star$ for the preferred semantics prf iff there exists a function $f^{\star}: \mathfrak{D}($ At $) \rightarrow \wp(\mathcal{V}($ At $) \times \mathcal{V}($ At $))$ that is imf-faithful w.r.t. prf s.t.:

$$
\operatorname{prf}(D \star \psi)=\min _{f^{\star}(D)}(\mathcal{V}(\psi))
$$

Remark 2. It can be shown that revision of formulas in $\mathcal{L}^{\mathrm{K}}$, i.e. operators of the type $\star: \mathcal{L}^{\mathrm{K}} \times \mathcal{L}^{\mathrm{K}} \rightarrow \mathcal{L}^{\mathrm{K}}$, are sound and complete w.r.t. faithful total preorders over the three-valued interpretations, completely analogous to the two-valued case (see Section 2.4). Thus, revision of ADFs equals revision of three-valued formulas plus realizability.

Remark 3. Kleene's logic is not the only logic for which the above characterisation result can be shown. In fact, careful inspection of the proof of Theorem 2 and 3 reveals that a similar characterisation result can be shown for any logic $L$ for a language $\mathcal{L}^{\mathrm{L}}(\mathrm{At})$ based on an interpretation function $\sigma_{\mathrm{L}}: \mathcal{L}^{\mathrm{L}}(\mathrm{At}) \rightarrow \mathcal{V}(\mathrm{At})$ for which the following properties hold:

1. $\sigma_{\mathrm{L}}^{\mathrm{L}}(\phi \wedge \psi)=\sigma_{\mathrm{L}}(\phi) \cap \sigma_{\mathrm{L}}(\psi)$ for any $\phi, \psi \in \mathcal{L}(\mathrm{At})$;

2. $\sigma_{\mathrm{L}}^{\mathrm{L}}(\phi \vee \psi)=\sigma_{\mathrm{L}}(\phi) \cup \sigma_{\mathrm{L}}(\psi)$ for any $\phi, \psi \in \mathcal{L}(\mathrm{At})$;

3. for every $v \in \mathcal{V}(\mathrm{At})$ there is some $\phi_{v} \in \mathcal{L}^{\mathrm{L}}(\mathrm{At})$ s.t. $\sigma_{\mathrm{L}}\left(\phi_{v}\right)=\{v\}$.

The results for grounded semantics that will be shown in the next section can likewise be adapted to other three-valued logics. The reason we used Kleene's three-valued logics in this paper is because it satisfies the above conditions and is a well-known and -studied logic for reasoning about undecidedness.

We now show how to overcome the problems described in Example 4 by refining the naive Dalal operator based on $\preceq_{D}^{\text {prf, } \Delta}$ :

Example 5. We define $\preceq_{D}^{\text {prf, } d+i}$ as a lexicographic combination of the information order and the order based on distance to preferred interpretations of the ADF $D$ under consideration. We first define the number of undecided nodes of an interpretation $v \in \mathcal{V}(\mathrm{At})$ as und $(v)=|\{s \in \mathrm{At} \mid v(s)=u\}|$. We now define (given two interpretations $v_{1}, v_{2} \in \mathcal{V}(S)$ ): $v_{1} \preceq_{D}^{\mathrm{prf}, \mathrm{d}+\mathrm{i}} v_{2}$ iff:

1. $v_{1} \in \operatorname{prf}(D)$, or

2. $v_{1}, v_{2} \notin \operatorname{prf}(D)$ and und $\left(v_{1}\right)<\operatorname{und}\left(v_{2}\right)$; or

\footnotetext{
${ }^{4}$ Due to spatial restrictions, we were not able to include all proofs.
}

3. $v_{1}, v_{2} \notin \operatorname{prf}(D)$ and und $\left(v_{1}\right) \quad \nless \quad$ und $\left(v_{2}\right)$ and $\min _{v \in \operatorname{prf}(D)}\left(v \Delta v_{1}\right) \leq \min _{v \in \operatorname{prf}(D)}\left(v \Delta v_{2}\right)$.

It can be showns that $\preceq$ prf, $\mathrm{d}+\mathrm{i}$ is a total preoder.

We illustrate this preorder with the ADF $D_{1}$ from Example 2. We get the following preorder on interpretations:

$$
\begin{aligned}
& \top \perp \top, \quad \perp \top \top \prec_{D}^{\text {prf }, d+i}
\end{aligned}
$$

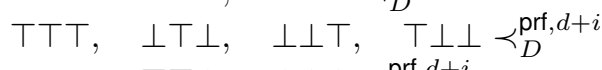

$$
\begin{aligned}
& \top \top \perp, \quad \perp \perp \perp \prec_{D}^{\text {prf }, d+i} \\
& \perp u \top, \quad \top u \top, \quad \perp \top u, \quad \top \perp u, \quad u \top \top, \quad u \perp \top \prec_{D}^{\text {prf }, d+i} \\
& \begin{array}{r}
u \perp \perp, \quad \top \top u, \quad \top u \perp, \quad \perp u \perp, \quad \perp \perp u, \quad u \top \perp \prec_{D}^{\text {prf, } d+i} \\
\perp u u, \quad u \top u, \quad u u \top, \quad u \perp u, \quad \top u u \prec_{D}^{\text {prf, } d+i}{ }_{D}
\end{array} \\
& \begin{aligned}
\perp u u, \quad u \top u, \quad u u \top, \quad u \perp u, \\
u u \perp \prec_{D}^{\operatorname{prf}, d+i}
\end{aligned} \\
& \text { uиu }
\end{aligned}
$$

We give two examples of revisions. First consider $D \star \sim b$ which has as preferred models $\operatorname{prf}(D \star \sim c)=$ $\{\perp \top \perp, \top \perp \perp\}$. Second, consider $D \star \odot b$ which has as preferred models $\operatorname{prf}(D \star \odot b)=\{\perp u \top, \top u \top\}$. Notice that a benefit of the approach to belief revision of ADFs presented in this section is that it is possible to revise by formulas having the third truth value $u$.

We now show with a second example that imf-mappings do not necessarily have to be refinements of the informationordering on interpretations.

Example 6. We can even reverse the second item of the definition of $\preceq_{D}^{\text {prf, } \mathrm{d}+\mathrm{i}}$ and still obtain an imf-mapping. We define $\preceq_{D}^{\text {prf, } \mathrm{d}+\mathrm{ri}}$ just like $v_{1} \preceq_{D}^{\text {prf, } \mathrm{d}+\mathrm{i}}$ in Example 5, but replace the third condition with: $v_{1}, v_{2} \notin \operatorname{prf}(D)$ and und $\left(v_{1}\right) \mathbb{L}_{i}$ $\operatorname{und}\left(v_{2}\right)$ and $\min _{v \in \operatorname{prf}(D)}\left(v \Delta v_{1}\right) \leq \min _{v \in \operatorname{prf}(D)}\left(v \Delta v_{2}\right)$. For ADF $D_{1}$ from Example 2 we then obtain the following $\preceq_{D}^{\text {prf, } \mathrm{d}+\mathrm{ri}}$-order on three-valued interpretations:

$$
\begin{aligned}
& \begin{array}{c}
\perp \top \top, \quad \top \perp\rfloor_{\mathrm{prfd}+\mathrm{ri}}^{\text {prfd }+\mathrm{ri}} \\
u u u \coprod_{D}
\end{array} \\
& \perp u u, \quad u \top u, \quad u u \top, \quad u \perp u, \quad \top u u \preceq_{D}^{\mathrm{prfd}+\mathrm{ri}} \\
& u u \perp \preceq_{D}^{\mathrm{prfd}+\mathrm{ri}} \\
& \top \perp u, \quad \perp u \top, \quad \top u \top, \quad \perp \top u, \quad u \perp \top, \quad u \top \top \preceq_{D}^{\text {prfd }+\mathrm{ri}} \\
& \begin{aligned}
u \top \perp, \quad \perp u \perp, & \perp \perp u, \quad \top \top u, \quad \top u \perp, \quad u \perp \perp \preceq_{D}^{\text {prfd }+\mathrm{ri}} \\
\top \top \top, & \perp \top \perp, \quad \perp \perp \top, \quad \top \perp \perp \preceq_{D}^{\text {prfd }+\mathrm{ri}}
\end{aligned} \\
& \top \top \perp, \quad \perp \perp \perp
\end{aligned}
$$

To illustrate the difference with $\preceq_{D}^{\text {prf, } \mathrm{d}+\mathrm{i}}$, observe that now $\operatorname{prf}(D \star \sim c)=\{u u u\} .^{5}$

\subsection{Revision of ADFs under Grounded Semantics}

In this section we characterise revisions under the grounded semantics by a class of total preorders. The basic idea is that every "layer" contains exactly one interpretation, which ensures that every $\preceq_{D}$-minimal set of interpretations is singleton and thus realizable under the grounded semantics.

\footnotetext{
${ }^{5}$ The revision operator from Examples 5 and 6 have been implemented in Java using the Tweety-library (Thimm 2017).
} 
Definition 8. Given a semantics Sem and an ADF $D=$ (At, $L, C$ ), a mapping $f: \mathfrak{D}(\mathrm{At}) \rightarrow \wp(\mathcal{V}(\mathrm{At}) \times \mathcal{V}(\mathrm{At}))$ associating a total preorder $\preceq_{D}$ to every ADF $D$ is an anti-symmetric faithful mapping (asf-mapping for semantics Sem if it is faithful w.r.t. Sem and for every $D \in \mathfrak{D}(\mathrm{At})$ and for every $v_{1}, v_{2} \in \mathcal{V}(\mathrm{At}):$ if $v_{1} \approx_{D} v_{2}$ then $v_{1}=v_{2}$.

We now show that $A D F_{\star}^{3}$-operator for the grounded semantics can be characterized by asf-mappings. The proof of this Theorem is analogous to the proof of Theorem 2, besides that realizability of $D \star \phi$ now corresponds to the anti-symmetry condition.

Theorem 3. An operator $\star: \mathfrak{D}(\mathrm{At}) \times \mathcal{L}^{\mathrm{K}}(\mathrm{At}) \rightarrow \mathcal{L}^{\mathrm{K}}(\mathrm{At})$ is an $A D F_{\star}^{3}$-operator for grounded iff there exists a function $f^{\star}: \mathfrak{D}(\mathrm{At}) \rightarrow \wp(\mathcal{V}(\mathrm{At}) \times \mathcal{V}(\mathrm{At}))$ that is asf-faithful w.r.t. grounded s.t.:

$$
\operatorname{grounded}(D \star \psi)=\min _{f^{\star}(D)}(\mathcal{V}(\psi))
$$

\section{Nonmonotonic Inference and Defeasible Conditionals for ADFs}

In this section, we study interrelations between $\mathrm{ADF}_{\star^{-}}^{3}$ operators, trivalent defeasible conditionals and nonmonotonic inference based on three-valued logic. We first define nonmonotonic inference based on three-valued logic and show how they can be equivalently viewed as trivalent defeasible conditionals. Thereafter, we define both static conditionals and dynamic conditionals for ADFs, which are defined using the Ramsey test on the basis of the revision operators defined and studied above. Finally, we show that the interrelations between revision, conditionals and inference relations known from propositional beliefs hold also in our argumentative setting.

\subsection{Three-valued Nonmonotonic Inference and Defeasible Conditionals}

Nonmonotonic inference on the basis of three-valued logics such as $\mathrm{K}$ can be defined completely analogously to the twovalued case, by specifying total preorders $\preceq$ that express a comparative measure of plausibility over the set of threevalued interpretations. We can then easily generalize the definition of conditional entailment to sets of three-valued interpretations. Given a set of atoms At, we assume a total preorders $\preceq$ over $\mathcal{V}(\mathrm{At})$. We can now define conditional entailment based on Kleene's three-valued logics as follows:

Definition 9. Given a set of atoms At, a total preorder $\preceq$ over $\mathcal{V}(\mathrm{At})$, and some $\phi, \psi \in \mathcal{L}^{\mathrm{K}}(\mathrm{At}), \phi \sim_{\preceq}^{\mathrm{K}} \psi$ iff $v \prec v^{\prime}$ for some $v \in \min _{\preceq}(\mathcal{V}(\phi \wedge \psi))$ and $v^{\prime} \in \min _{\preceq}(\mathcal{V}(\phi \wedge \sim \psi)){ }^{6}$

Notice the choice of negation in the definition above. This is to ensure that an inference $\phi \sim_{\prec}{ }_{\prec} \psi$ is valid iff all $\preceq$ minimal worlds that validate $\phi$ validate $\psi$. That using $\sim$ in the above definition of conditional inference ensures this is shown by the following fact:

\footnotetext{
${ }^{6}$ Since $\preceq$ is a total order, we can equivalently replace any of the two existential quantifiers expressed by "for some" by a universal quantifier.
}

Fact 4. $\phi \sim_{\preceq}^{\mathrm{K}} \psi$ iff $\min _{\preceq} \mathcal{V}(\phi) \subseteq \mathcal{V}(\psi)$.

This fact shows that, just like in the case of classical nonmonotonic inference relations, three-valued nonmonotonic inference relations obtained on the basis of a total preorder can be equivalently viewed as conditional inference relations on the basis of the selection function $\min _{\preceq}$. In other words, conditionals $(\psi \mid \phi)$, defined on the basis of the selection function $\min \preceq$ can be simply seen as the syntactic counterparts of the nonmonotonic inference relation $\sim_{\prec}^{K}$.

We show that any inference relation based on a total preorder over $\mathcal{V}(\mathrm{At})$ satisfies (REF), (CUT), (CM), (RW), (LLE), (OR) and a postulate we call weak Rational Monotony (wRM):

(wRM) $\phi \sim \gamma$ and $\phi K \sim \psi$ implies $\phi \wedge \psi \sim \gamma$ In the context of three-valued logics, the difference between (RM) and (wRM) is the following: the antecendent of (wRM) requires that from $\phi$, neither $\odot \psi$ nor $\neg \psi$ can be derived, i.e. if $\phi$ then normally $\psi$ is neither false nor undecided. (RM), on the other hand, has a weaker antecedent, namely that $\neg \psi$ cannot be derived, i.e. normally $\psi$ is not false if $\phi$ is accepted.

Analogously to Proposition 1, nonmonotonic inference relations induced by total preorders over three-valued interpretation satisfies all the KLM-postulates as well as the non-Horn postulate (wRM):

Proposition 4. Given a set of atoms At and a total preorder $\preceq$ over $\mathcal{V}(\mathrm{At}), \sim_{\prec}^{\mathrm{K}}$ satisfies (REF), (CUT), (CM), (RW), (LLE), (OR) and (wRM).

We show now that there are total preorders for which (RM) might be violated:

Example 7. Consider a preorder $\preceq$ over $\mathcal{V}(\{a, b\})$ s.t. $\top u \prec$ T丁. Then $a \sim^{\mathrm{K}} \odot b$ and $a K^{K} \stackrel{\mathrm{K}}{\complement} b$ yet $a \wedge b K_{\preceq}^{\mathrm{K}} \odot b$.

Altogether, we can conclude that the basic ideas for obtaining nonmonotonic conditional inferences and defeasible inference relations known from propositional logic can be taken over to the three-valued setting, but some subtle differences (e.g. (wRM) vs (RM)) distinguish the resulting inference relations from their two-valued counterparts.

\subsection{Defeasible Conditional Inference for ADFs}

In this section we study various ways of obtaining conditional inference relations on the basis of ADFs, and relate these conditional inference relations to revision and defeasible inference relations.

We first define static conditional inference relations, which treat the interpretations selected by some semantics given an ADF as equally plausible, and any other interpretation as implausible or even impossible. An ADF $D$ therefore implies a static conditional $\phi \Rightarrow \psi$ (given some semantics Sem), if there is an interpretation in $\operatorname{Sem}(D)$ that validates $\phi$, and every interpretation in $\operatorname{Sem}(D)$ that validates $\phi$ also validates $\psi$.

Definition 10. Let an ADF $D=($ At $, L, C)$, some semantics Sem and some $\phi, \psi \in \mathcal{L}^{\mathrm{K}}(\mathrm{At})$ be given. $D \sim_{\mathrm{Sem}}^{\mathrm{st}} \phi \Rightarrow \psi$ iff: 
- there is some $v \in \operatorname{Sem}(D)$ s.t. $v(\phi)=\top$, and

- for every $v \in \operatorname{Sem}(D)$ s.t. $v(\phi)=\top, v(\psi)=\top$.

Example 8. Consider again $D_{1}$ from Example 2. We have e.g. $D_{1} \sim_{\text {prf }}^{\text {st }} \top \Rightarrow c, \neg b \Rightarrow a, \neg a \Rightarrow b$.

Remark 4. In a two-valued propositional setting, static conditionals can be defined as follows: $\delta \sim^{\text {st }} \phi \Rightarrow \psi$ iff $\delta \nvdash \neg \phi$ (i.e. $\Omega(\delta) \cap \Omega(\phi) \neq \emptyset$ ) and $\delta \vdash \phi \rightarrow \psi$ (or equivalently: $\delta \wedge \phi \vdash \psi)$.

Static conditional inference relations, however, are rather weak, since their antecedents are restricted to formulas that are implied by at least one interpretation selected by Sem. For example, $D_{1} K_{\text {prf }}^{\text {st }} \neg c \Rightarrow \phi$ for any $\phi \in \mathcal{L}^{\mathrm{K}}(\{a, b, c\})$, not even $\neg c \Rightarrow \top$. Therefore, we introduce now dynamic conditional inference relations, based on revisions of ADFs. We construct a conditional inference relation for ADFs based on the Ramsey test, going back to (Ramsey 1931):

If two people are arguing "If $p$, then $q$ ?" and are both in doubt as to $p$, they are adding $p$ hypothetically to their stock of knowledge and arguing on that basis about $q$;

Based on this idea, we can simply state that the conditional $(\psi \mid \phi)$ is derivable from the ADF $D$, or, equivalently (in view of Fact 4) the conditional $(\psi \mid \phi)$ is valid in view of $D$, given a semantics Sem and some revision operator $\star$ iff $\psi$ is derivable in the revised ADF $D \star \phi$ under the semantics Sem:

Definition 11. Given an ADF $D$ and a revision operator $\star$, $D \sim_{\text {Sem }}^{\star}(\psi \mid \phi)$ iff $D \star \phi \sim_{\text {Sem }}^{\cap} \psi$.

We can show that static conditional inference relations are weaker than dynamic conditional inference relations, according to any $\mathrm{ADF}_{\star}^{3}$-operator:

Proposition 5. Let an ADF $D$, some semantics Sem and an $\mathrm{ADF}_{*}^{3}$-revision operator (for the semantics Sem) be given. Then $D \sim_{\text {Sem }}^{\text {st }} \phi \Rightarrow \psi$ implies $D \sim_{\text {Sem }}^{\star}(\psi \mid \phi)$.

We first show that dynamic conditional inference relations based on revision of ADFs can be seen as a special case of three-valued conditional inference relations. We do this by showing that, given a $\mathrm{ADF}_{\star}^{3}$-operator $\star$, the corresponding total preorder $f^{\star}(D)$ gives rise to an inference relation $\sim_{f^{\star}(D)}^{K}$ equivalent to the conditionals $\sim_{\text {Sem }}^{\star}$-derivable from $D$.

Proposition 6. Given an ADF $D$, some semantics Sem $\in\{$ prf, grounded $\}$ and an $A D F_{\star}^{3}$-operator $\star$ satisfying $\left(\mathrm{ADF}_{\star}^{3} 1\right)-\left(\mathrm{ADF}_{\star}^{3} 6\right), D \sim_{\text {Sem }}^{\star}(\psi \mid \phi)$ iff $\phi \sim_{f^{\star}(D)}^{\mathrm{K}} \psi$.

From this connection between dynamic conditionals and three-valued nonmonotonic inference relations, we can show that dynamic conditionals (or their equivalent formulation as nonmonotonic inference relations) satisfy all the KLM-postulates and (wRM):

Corollary 1. Let an ADF $D$, some semantics Sem $\in$ \{prf, grounded $\}$ and a $A D F_{\star}^{3}$-operator $\star$ for Sem be given. Then $\sim_{f^{\star}(D)}^{K}$ satisfies (REF), (CUT), (CM), (RW), (LLE), (OR) and (wRM).

We illustrate these conditional inference relations with some conditionals derived from Example 5:
Example 9 (Example 5 continued). Where $\star$ is the operator based on $\preceq_{D}^{\text {prf, } \mathrm{d}+\mathrm{i}}$ and $D_{1}$ is as in Example 5, we see that e.g. $\sim c \sim \sim_{D_{1}, \star}^{\text {Sem }} \neg c$ and $a \sim{ }_{D_{1}, \star}^{\text {Sem }} \neg a \wedge c$. Notice that also e.g. $c \sim{ }_{D_{1}, \star}^{\text {Sem }} C_{c}$ (i.e. $c \sim \sim_{D_{1}, \star}^{\text {Sem }} \neg a \vee \neg b$ ) and $C_{c} \sim{ }_{D_{1}, \star}^{\text {Sem }} c$. In fact for any $s \in\{a, b, c\}, s \sim{ }_{D_{1}, \star}^{\text {Sem }} C_{s}$ and $C_{s} \sim \sim_{D_{1}, \star}^{\text {Sem }} s$.

The syntactical structure of an ADF is not always preserved by the dynamic conditional inference relation:

Example 10. Let $D=\left(\{a\}, L, C_{a}=\neg a\right)$ and consider the preorder $u \prec T \prec \perp$. It can be easily shown that there exists an i-modular mapping $f$ s.t. $f(D)=\prec$. However, $a K_{D, \star}^{\text {Sem }} \neg a$ and $\neg a \underset{D, \star}{\text { Sem }_{D, \star}} a$. A similar, but more involving example without a self-attacking argument for which a similar claim holds is: $D^{\prime}=\left(\{a, b, c\}, L, C_{a}=\neg b, C_{b}=\right.$ $\left.\neg c, C_{c}=\neg a\right)$.

\section{Related Works}

To the best of our knowledge, no papers detailing conditional inference from argumentative formalisms on the basis of the Ramsey test have been published before. we discuss related works that treat either revision or conditional inference in argumentative formalisms.

Revision of ADFs is investigated in (Linsbichler and Woltran 2016a), where revision of ADFs by other ADFs are defined. Conceptually, our approach is able to capture the approach by (Linsbichler and Woltran 2016a) since we allow for revisions of ADFs under three-valued semantics by any $\mathcal{L}^{\mathrm{K}}$, which allows to express revision by a set of interpretations $\mathcal{V}^{\prime}$ as revision by the formula $\bigvee \mathcal{V}^{\prime}$. Technically, there is some incomparability between our approach and that of (Linsbichler and Woltran 2016a) caused by the difference in the type of revision we consider. In particular, there are differences in the way the issue of realizability is handled. We have chosen to handle this issue by ensuring that any subset of a ${ }_{D}$-layer is realizable under a given semantics, whereas (Linsbichler and Woltran 2016a) handles this issue by defining revisions of the ADF $D$ by another ADF as $f_{\text {Sem }}\left(\min _{\preceq_{D}} \operatorname{Sem}(F)\right)$, where the function $f_{\text {Sem }}\left(\mathcal{V}^{\prime}\right)$ returns $\mathcal{V}^{\prime}$ if it is realizable under $\mathcal{V}^{\prime}$ and the interpretation $v_{u}{ }^{7}$ otherwise.

Revisions of abstract argumentation are considered in many works, including (Cayrol, de St-Cyr, and LagasquieSchiex 2008; Falappa, Kern-Isberner, and Simari 2009; Coste-Marquis et al. 2014; Baumann and Brewka 2015; Linsbichler and Woltran 2016b; Delobelle, Konieczny, and Vesic 2015; Delobelle et al. 2016; Mailly 2015; Linsbichler 2017). In (Diller et al. 2018), revisions of argumentation frameworks by both propositional formulas and other argumentation frameworks (represented as sets of extensions according to some semantics) are defined indirectly by specifying the set of extensions (according to some semantics). Thus, conceptually, we provide generalisations of both these kinds of revisions, as we allow for revisions by any formula in the language $\mathcal{L}^{\mathrm{K}}$, which allows to represent sets of extensions. With regards to the differences between revision

\footnotetext{
${ }^{7}$ Recall that $v_{u}(s)=u$ for every $s \in$ At.
} 
of abstract argumentation frameworks and abstract dialectical frameworks, we conjecture that we can characterise revisions of abstract argumentation frameworks as revisions of ADFs. (Coste-Marquis et al. 2014) allow for revision of sets of argumentation frameworks by propositional formulas, resulting in a set of argumentation frameworks. The postulates governing such revisions are adaptions of the AGMpostulates similar to both ours and those of (Diller et al. 2018), even though both (Diller et al. 2018) and we consider revisions of single abstract argumentation resp. dialectical frameworks. Restricting attention to single frameworks has as an effect that realizability is an essential concern, which (Coste-Marquis et al. 2014) avoid. Furthermore, we are more general in the sense we study revision of ADFs, which can capture argumentation frameworks, by formulas in $\mathcal{L}^{\mathrm{K}}$, which includes propositional formulas as a special case.

A number of works have studied the conditional inferential behaviour of formal argumentation formalisms. In structured argumentation, there are a number of works that study KLM-like properties of argumentative inference relations (Borg, Straßer, and Arieli 2020; Heyninck and Arieli 2018; Heyninck and Straßer 2020; Čyras and Toni 2015; Cyras and Toni 2016; Li, Oren, and Parsons 2017). These work differ both in host formalism (various formalisms for structured argumentation versus ADFs) and the way conditional inference is defined. Whereas we define conditional inference using the Ramsey-test, these works consider a conditional $(\psi \mid \phi)$ to be justified if, after addition of $\phi$ to the knowledge base (sometimes as a strict premise, sometimes as a defeasible premise), $\psi$ is derivable according to the chosen argumentative inference relation. These works do not agree on how exactly $\phi$ is added, i.e. in some works it is added as a defeasible premise whereas in other works it is added as a strict premise.

In (Rienstra 2014, Chapter 3) a type of entailment for abstract argumentation frameworks is defined which has similarities to the work done in our paper. Entailments are based on interventions of argumentation frameworks, inspired by interventions in Bayesian networks. Interventions of argumentation frameworks allow to enforce a labelling status of an argument by adding new arguments that attack the argument whose labelling status is to be intervened. Given an argumentation framework $F$, an entailment relation based on such interventions is then defined by stating that $\Psi \sim{ }_{\sigma}^{F} \phi$ iff after the intervention $\Psi, \phi$ is true according to all $\sigma$ labellings of the argumentation framework that is the result of the intervention $\Psi$ on $F$. (Rienstra 2014) studies several properties of such inference relations, include the KLMproperties, for which it is shown that for restricted classes of interventions, some semantics satisfy cautious monotony, cut and rational monotony. Another approach is that of conditional acceptance functions (Booth et al. 2012), where, given an argumentation framework, the usual labelling semantics are changed as to account for abductive or counterfactual reasoning. In (Booth et al. 2013) conditional inference relations for abstract argumentation frameworks are defined on the basis of a propositional language built up from atoms $\mathrm{in}_{s}$, out ${ }_{s}$ and $\mathrm{u}_{s}$ for every argument $s$, which encode argument labels. Nonmonotonic inference relations are then defined semantically by a total preorder over models for this language by preferring models that model labellings that "satisfy better" the constraints of a selected semantics (given the argumentation framework under consideration). Our work takes a different approach to the definition of conditional inference relation by using the Ramsey-test, and is more general since ADFs subsume abstract argumentation.

\section{Conclusion}

In this paper we defined dynamic conditional inference relations for ADFs based on the Ramsey test, and developed a new approach to revision of an ADF by formulas to achieve this. We have shown that such conditional inference relations satisfy all the usual rationality postulates for conditional inferences and extend static conditionals but also give rise to subtle differences with the propositional case, as witnessed e.g. by the (wRM)-postulate. What comes out clearly from this work is that revision, or more generally belief change, is the platform that allowed us to bridge the gap between argumentative reasoning and conditional inference. As such, we hope that this work will serve as an inspiration for further investigations into the combination and crossfertilization between argumentative and nonmonotonic conditional reasoning. Indeed, rather than a definitive statement on dynamic conditional inference for ADFs, we see this paper as an anchor point for further research on revision, nonmonotonic inference and dynamic conditional argumentative reasoning. When generalizing these interconnected concepts, there are many choices to be made, such as which "monotonic base logic" to use (in our case: K), which postulates for revision to use (e.g. the approach of (Katsuno and Mendelzon 1991) vs alternative types of revision (Fermé and Hansson 2018)), how exactly to adapt the postulates for revision and the corresponding faithful mappings (e.g. equivalence vs strong equivalence). Even though the choices we made are well-motivated, they are clearly not the only viable ones. It remains to be seen whether the interconnections between revision, inference and conditionals generalize to such a setting.

In future work, we plan to study the impact of changes in the choices made in this paper as outlined above, as well as look at more specific revision operators (Kern-Isberner 2001). Furthermore, we plan to look deeper into the semantical nature of revisions defined in this work. Indeed, a revised ADF is only defined in terms of its models (according to a chosen semantics). What is not specified is how we can obtain the revised ADF in terms of changes (be it revisions or otherwise) of the original ADF, and in particular its conditions. We plan to investigate how this semantical perspective can be supplemented with a characterisation of revision of ADFs in terms of change of the acceptance conditions of the revised ADF. For such investigations, iterated revision (Kern-Isberner 2004; Darwiche and Pearl 1997) might prove relevant, due to its well-established connections with conditional change. Finally, we will investigate the complexity of the revision operators from Example 5. 


\section{Acknowledgements}

The research reported here was supported by the Deutsche Forschungsgemeinschaft under grant KE 1413/11-1.

\section{References}

Alchourrón, C. E.; Gärdenfors, P.; and Makinson, D. 1985. On the logic of theory change: Partial meet contraction and revision functions. Journal of symbolic logic 510-530.

Baumann, R., and Brewka, G. 2015. AGM meets abstract argumentation: Expansion and revision for Dung frameworks. In Proceedings of the 24th International Joint Conference on Artificial Intelligence (IJCAI'15).

Bochvar, D. A., and Bergmann, M. 1981. On a threevalued logical calculus and its application to the analysis of the paradoxes of the classical extended functional calculus. History and Philosophy of Logic 2(1-2):87-112.

Booth, R.; Kaci, S.; Rienstra, T.; and van der Torre, L. W. N. 2012. Conditional acceptance functions. In Verheij, B.; Szeider, S.; and Woltran, S., eds., Computational Models of Argument - Proceedings of COMMA 2012, Vienna, Austria, September 10-12, 2012, volume 245 of Frontiers in Artificial Intelligence and Applications, 470-477. IOS Press.

Booth, R.; Kaci, S.; Rienstra, T.; and van der Torre, L. W. N. 2013. Monotonic and nonmonotonic inference for abstract argumentation. In Boonthum-Denecke, C., and Youngblood, G. M., eds., Proceedings of the Twenty-Sixth International Florida Artificial Intelligence Research Society Conference, FLAIRS 2013, St. Pete Beach, Florida, USA, May 22-24, 2013. AAAI Press.

Borg, A.; Straßer, C.; and Arieli, O. 2020. A generalized proof-theoretic approach to structured argumentation by hypersequent calculi. Studia Logica. Accepted. https://doi.org/10.1007/s11225-020-09906-1.

Brewka, G.; Strass, H.; Ellmauthaler, S.; Wallner, J. P.; and Woltran, S. 2013. Abstract dialectical frameworks revisited. In ICJAI.

Cayrol, C.; de St-Cyr, F. D.; and Lagasquie-Schiex, M.-C. 2008. Revision of an argumentation system. In Brewka, G., and Lang, J., eds., Proceedings of the Eleventh International Conference on Principles of Knowledge Representation and Reasoning (KR'2008), 124-134. Menlo Park, California, USA: AAAI Press.

Coste-Marquis, S.; Konieczny, S.; Mailly, J.-G.; and Marquis, P. 2014. On the revision of argumentation systems: Minimal change of arguments statuses. In Proceedings of the 14th International Conference on Principles of Knowledge Representation and Reasoning (KR'14).

Čyras, K., and Toni, F. 2015. Non-monotonic inference properties for assumption-based argumentation. In Black, E.; Modgil, S.; and Oren, N., eds., Proceedings of the 3rd International Workshop on Theory and Applications of Formal Argument (TAFA'15), volume 9524 of Lecture Notes in Computer Science, 92-111. Springer.

Cyras, K., and Toni, F. 2016. Properties of ABA+ for non-monotonic reasoning. In 6th International Workshop on Non-Monotonic Reasoning (NMR), Cape Town, 25-34.
Dalal, M. 1988. Investigations into a theory of knowledge base revision: preliminary report. In Proceedings of the Seventh National Conference on Artificial Intelligence, volume 2, 475-479. Citeseer.

Darwiche, A., and Pearl, J. 1997. On the logic of iterated belief revision. Artificial Intelligence 89(1-2):1-29.

Delgrande, J. P., and Peppas, P. 2015. Belief revision in horn theories. Artificial Intelligence 218:1-22.

Delobelle, J.; Haret, A.; Konieczny, S.; Mailly, J.-G.; Rossit, J.; and Woltran, S. 2016. Merging of abstract argumentation frameworks. In Proceedings of the 15th International Conference on Principles of Knowledge Representation and Reasoning (KR'16).

Delobelle, J.; Konieczny, S.; and Vesic, S. 2015. On the aggregation of argumentation frameworks. In Proceedings of the Twenty-Fourth International Joint Conference on Artificial Intelligence (IJCAI 2015), 2911-2917.

Diller, M.; Haret, A.; Linsbichler, T.; Rümmele, S.; and Woltran, S. 2018. An extension-based approach to belief revision in abstract argumentation. International Journal of Approximate Reasoning 93:395-423.

Falappa, M. A.; Kern-Isberner, G.; and Simari, G. R. 2009. Belief revision and argumentation theory. In Argumentation in Artificial Intelligence. Springer. 341-360.

Fermé, E., and Hansson, S. O. 2018. Belief change: introduction and overview. Springer.

Gärdenfors, P. 1986. Belief revisions and the ramsey test for conditionals. The Philosophical Review 95(1):81-93.

Gärdenfors, P. 1990. Belief revision and nonmonotonic logic: two sides of the same coin? In European Workshop on Logics in Artificial Intelligence, 52-54. Springer.

Hansson, S. O. 1999. A survey of non-prioritized belief revision. Erkenntnis 50(2):413-427.

Hawthorne, J. 2007. Nonmonotonic conditionals that behave like conditional probabilities above a threshold. Journal of Applied Logic 5(4):625-637.

Heyninck, J., and Arieli, O. 2018. On the semantics of simple contrapositive assumption-based argumentation frameworks. In Modgil, S.; Budzynska, K.; and Lawrence, J., eds., Proceedings of the 7th International Conference on Computation Models of Argument (COMMA'18), Frontiers in Artificial Intelligence and Applications 305, 9-20. IOS Press.

Heyninck, J., and Straßer, C. 2020. A comparative study of assumption-based approaches to reasoning with priorities. Forthcoming.

Heyninck, J. 2019. Relations between assumption-based approaches in non-monotonic logic and formal argumentation. Journal of Applied Logics 6(2):317-357.

Katsuno, H., and Mendelzon, A. O. 1991. Propositional knowledge base revision and minimal change. Artificial Intelligence 52(3):263-294.

Kern-Isberner, G., and Simari, G. R. 2011. A default logical semantics for defeasible argumentation. In FLAIRS 24.

Kern-Isberner, G. 2001. Conditionals in nonmonotonic 
reasoning and belief revision: considering conditionals as agents. Springer-Verlag.

Kern-Isberner, G. 2004. A thorough axiomatization of a principle of conditional preservation in belief revision. Annals of Mathematics and Artificial intelligence 40(1):127164.

Kraus, S.; Lehmann, D.; and Magidor, M. 1990. Nonmonotonic reasoning, preferential models and cumulative logics. AI 44(1-2):167-207.

Li, Z.; Oren, N.; and Parsons, S. 2017. On the links between argumentation-based reasoning and nonmonotonic reasoning. In $T A F A, 67-85$. Springer.

Linsbichler, T., and Woltran, S. 2016a. Revision of abstract dialectical frameworks: Preliminary report. In First International Workshop on Argumentation in Logic Programming and Non-Monotonic Reasoning, Arg-LPNMR 2016.

Linsbichler, T., and Woltran, S. 2016b. Revision of abstract dialectical frameworks: Preliminary report. In Gaggl, S.; Nieves, J. C.; and Strass, H., eds., First international Workshop on Argumentation in Logic Programming and NonMonotonic Reasoning, Arg-LPNMR 2016.

Linsbichler, T. 2017. Advances in Abstract Argumentation: Expressiveness and Dynamics. Ph.D. Dissertation, TU Wien.

Mailly, J.-G. 2015. Dynamics of Argumentation Frameworks. Ph.D. Dissertation, Université d'Artois.

Makinson, D., and Gärdenfors, P. 1991. Relations between the logic of theory change and nonmonotonic logic. In The logic of theory change. Springer. 183-205.

Makinson, D. 1988. General theory of cumulative inference. In International Workshop on Non-Monotonic Reasoning (NMR), 1-18. Springer.

Makinson, D. 1993. Five faces of minimality. Studia Logica 52(3):339-379.

Makinson, D. 2011. Conditional probability in the light of qualitative belief change. Journal of Philosophical Logic 40(2):121-153.

Nute, D. 1984. Conditional logic. In Handbook of philosophical logic. Springer. 387-439.

Pührer, J. 2020. Realizability of three-valued semantics for abstract dialectical frameworks. Artificial Intelligence 278:103198.

Ramsey, F. P. 1931. General propositions and causality. Foundations of Mathematics.

Rienstra, T. 2014. Argumentation in flux: modelling change in the theory of argumentation. Ph.D. Dissertation, University of Luxembourg, Luxembourg.

Shoham, Y. 1987. A semantical approach to nonmonotonic logics. In Readings in nonmonotonic reasoning, 227-250.

Strass, H. 2014. On the relative expressiveness of argumentation frameworks, normal logic programs and abstract dialectical frameworks. In 15TH International Workshop on Non-Monotonic Reasoning (NMRM 2014, Vienna, Austria, 292. Citeseer.
Thimm, M., and Kern-Isberner, G. 2008. On the relationship of defeasible argumentation and answer set programming. COMMA 8:393-404.

Thimm, M. 2017. The tweety library collection for logical aspects of artificial intelligence and knowledge representation. KI-Künstliche Intelligenz 31(1):93-97.

Urquhart, A. 2001. Basic many-valued logic. In Handbook of philosophical logic. Springer. 249-295.

Varzi, A. C., and Warglien, M. 2003. The geometry of negation. Journal of Applied Non-Classical Logics 13(1):919. 